\title{
Research on Vocational College Course Evaluation Based on Data Mining
}

\author{
Hui Zhang \\ Electronic and Electrical Department, Wuhan Railway Vocational College of Technology, Wuhan, \\ 430205, China, 407706483@qq.com
}

\begin{abstract}
Keywords: Course Management, vocational college course evaluation, BP neural network, Levenberg-Marqardt optimization algorithm.
\end{abstract}

\begin{abstract}
Implementing course evaluation is an effective way to enhance vocational college course management level, and how to evaluate vocational college course is one of the difficulties and hot research fields for the researchers related. The paper takes science and engineering major course for example and presents a new model for evaluating vocational college course evaluation based on data mining technology. First an evaluation indicator system of vocational college course evaluation with three grades is designed; Second, aiming at the shortages of the existing BP neural network algorithm of data-mining for evaluating vocational college course performance, Levenberg-Marqardt optimization algorithm is used to correct BP neural network to speed up the convergence and simplify the structure and to improve evaluating accuracy of the original BP model. Finally the model is realized with the data from three vocational colleges and the realization of the experimental results show that the model can improve algorithm efficiency and evaluation accuracy and can be used for vocational college course evaluation practically.
\end{abstract}

\section{Introduction}

Vocational colleges shoulder the divine mission of training the successors with high quality and high esthetic sentiment. To strengthen the training of musicianship and quality of vocational college students is the modernized, technological and humanized demand of education. As an important subject of art education, music is considered to an important subject, is the contents of life-long education, is the education contents of the entire society, and is the education contents of schools, especially institutions of higher learning, a holy place for the emergence of talents. Hence, study on the education quality evaluation of vocational college course has also become one of the research hotspots in the industry, the research contents of which include the study on evaluation system of vocational college course and evaluation method. This thesis will take scientific and technical majors course evaluation as example to carry out the study of indicator system and calculation method for vocational colleges course teaching evaluation [1, 2].

As for evaluation methods, Analytic hierarchy process, Fuzzy comprehensive evaluation, Data Mining evaluation are three mainstream methods and in which BP neural network evaluation, as a typical data mining method, is most welcomed by the most researchers for its high evaluation accuracy and powerful data mining ability. But BP neural network algorithm is easy to be trapped into defects like local minimum, over-learning, strong operation specialization which limited practical uses in engineering evaluation $[3,4,5]$.

In the specific evaluation process of vocational college teaching, this thesis, as for evaluation contents, mainly focuses on teaching management, course construction, teaching conditions, teaching process, teachers and teaching effects; as for evaluation principles, launching from such four aspects as experts' evaluation, internal evaluation, self-evaluation and social evaluation, obtaining evaluation data by adopting the above principles as for the data selection of specific evaluation indicators; as for evaluation method, BP neural network algorithm of data mining is improved to overcome the question of slow convergence speed of BPNN. In so doing, not only the problem of convergence speed of BPNN has been solved, but also the simplicity of the model structure and the accuracy of the evaluation are ensured. 


\section{Evaluation Indicator System Design}

In order to improve the scientificity of vocational college course evaluation, we shall embody the abstract evaluation objectives. Indicators are one of the stipulations of objectives; they are specific, measurable and operable objectives. Only the indicator system formed by several specific indicators of systemization and close connection can reflect the entire objective, reduce the possibility of evaluation discrepancy caused by the difference of evaluators' level, perspective and impression, decrease the subjectivity of evaluation, and increase objectivity. It is thus clear that the establishment of evaluation indicator system is the key to guarantee the unified criteria, justice and objectivity of evaluation $[6,7,8]$. Therefore, this thesis, while establishing vocational college course evaluation indicator system, focuses on such principles for choosing indicators as conformity to teaching objectives, direct measurability and comparability of objectives and an evaluation indicator system is constructed with 6 first-class indicators which are teaching management, course construction, teaching conditions, teaching process, teachers, teaching effects respectively and 12 second-class indicators, 25 third-class indicators according to practical situation as the observation point of evaluation. Limited to length, specific indicators are no longer described in detail.

\section{Evaluation Algorithm Design}

\subsection{BP Neural Network Structure}

BP neural network is generally comprised of input layer, hidden layer and output layer, each layer connecting to the other, the node of each layer not connecting. The number of nodes of input layer generally adopts the dimension of input vector, and that of output layer generally adopts the dimension of output vector; there has no certain standard to obtain the number of nodes of hidden layer which shall be obtained through repeated cut-and-try methods. According to Kolmogorv law, three-layer BP neural network with one hidden layer (sufficient nodes of hidden layer) is able to approximate any nonlinear continuous function in any accuracy on a closed set. Therefore, this thesis adopts BP neural network with single hidden layer.

Suppose that the input vector of $\mathrm{X}$ is $x \in R^{n}$, in which $x=\left(x_{0}, x_{1}, x_{2} \ldots, x_{n-1}\right)^{T}$; there are $n_{1}$ neurons in the hidden layer, the output of which is $x^{\prime} \in R^{n 1}, x^{\prime}=\left(x_{0}^{\prime}, x_{1}^{\prime}, x_{1_{2}} \ldots, x_{n-1}^{\prime}\right)^{T}$; there are $m$ neurons in the output layer, output $y \in R^{m}, y=\left(y_{0}, y_{1}, y_{2} \ldots, y_{n-1}\right)^{T}$, the weight from input layer to hidden layer is $w_{i, j}$, threshold is $\theta_{j}$; the weight from hidden layer to output layer is $w_{j, k}$, threshold is $\theta_{k}^{\prime}$; hence, output of neurons in each layer is as shown in Formula 1[4].

$$
\left\{\begin{array}{c}
x_{j}^{\prime}=f\left(\sum_{i=0}^{n-1} w_{i j} x_{i}-\theta_{j}\right), \quad j=0,1,2 \ldots, n_{1}-1 \\
y_{k}^{\prime}=f\left(\sum_{i=0}^{n 1-1} w_{j k}^{\prime} x_{j}^{\prime}-\theta_{k}^{\prime}\right), \quad k=0,1,2 \ldots, m-1
\end{array}\right.
$$

Obviously, it will complete the mapping from $n$ dimensional space vector to $m$ dimension, in which activation function $f(x)$ is unipolar. Sigmoid function is as shown in Formula 2. $f(x)$ is continuous differentiable and meets Formula 3[5].

$$
\begin{array}{r}
f(x)=\frac{1}{1+e^{-x}} \\
f^{\prime}(x)=f(x)(1-f(x))
\end{array}
$$

\subsection{Basic Idea and Step to Improv BP Algorithm}

Traditional BP algorithm adopts the fastest grads descent method to revise weight; training process is to start from certain point, along the slope of error function, gradually reaching minimum point and making the error be zero. However, as for complicated network, error curved surface is like 
a bowl in multi-dimensional space and the bottom of the bowl is the minimum point. But the surface of the bowl is uneven, so it may fall into certain local minimum point in training process, changing towards multi directions from the point will increase the error, resulting in the failure of escaping from the local minimum point. This thesis, in allusion to the characteristics of non-linear system, improves the traditional BP network, introducing Levenberg-Marqardt optimization algorithm. Its basic idea is to make the iterations no long towards single negative gradient direction, and permits the error to carry out search along the deterioration direction, meanwhile, optimize network weight through the adaptive adjustment between the fastest grads descent method and Gauss-Newton method, making the network able to effectively converge, greatly improving the convergence rate and generalization ability of network.

L-M optimization algorithm, also called damped least square method, the weight adjustment is Formula 4, in which $e$ is error vector, $J$ is the Jacobi matrix of error to weight differential, $\mu$ is a scalar; while $\mu$ is increasing, it is close to the fastest descent method with small learning rate; while $\mu$ is decreasing to 0 , the algorithm becomes Gauss-Newton method. So $\mathrm{L}-\mathrm{M}$ algorithm is the smooth reconciliation between the fastest grads decent method and Gauss-Newton method. The specific iterative step of $\mathrm{L}-\mathrm{M}$ algorithm is the following calculation process.

$$
\Delta \omega=\left(J^{T} J+\mu I\right)^{-1} J^{T} e
$$

Send all the inputs in network and calculate the output of network with Formula 5 and Formula 6. And calculate the error sum of squares of training and integrating all the targets with error function Formula 7. In Formula 2, Formula 3 and Formula 4, $\gamma_{h}^{k}$ represents the node output of hidden layer, $z_{j}^{k}$ represents outputting node output, $E$ is error function, ${ }^{X_{i}}$ is input signal, $\theta_{h}$ is the threshold of node of hidden layer $h, f$ is transfer function, $\omega$ is connection weight, $p$ is number of samples, and $k=1,2, \ldots P$.

$$
\begin{gathered}
\gamma_{h}^{k}=f\left(\sum_{i=1}^{N} \omega_{i h} \bullet x_{i}^{k}+\theta_{k}\right) \\
z_{j}^{k}=f\left(\sum_{h=1}^{N} \omega_{h j} \bullet \gamma_{h}^{k}+\gamma_{i}\right)=f\left[\sum_{h=1}^{N} \omega_{h j} \bullet \sum_{i=1}^{N} \omega_{i h} \bullet x_{i}^{k}+\theta_{h}\right)+\gamma_{j} \\
E=\frac{1}{2} \sum_{k, j}^{P, N}\left(T_{j}^{k}-z_{j}^{k}\right)^{2}
\end{gathered}
$$

(2) Calculate the Jacobi matrix $J$ of error to weight differential

Define the Marqardt sensitivity as Formula 8, in which $S_{i}^{m}$ is the sensitivity of the change of the $i$ th element input in $m$ layer, in which $n$ is the weight sum of network of each layer. The recurrence formula of sensitivity is Formula 9[3].

$$
\begin{gathered}
S_{i}^{m}=\frac{\partial E}{\partial n_{i}^{m}} \\
S_{p}^{m}=E\left(n_{q}^{m}\right)\left(\omega^{m+1}\right)^{T} S_{q}^{m+1}
\end{gathered}
$$

It is thus clear that sensitivity can be reversely spread from the first layer to the last layer through network, as shown in Formula 10. Finally, calculate the elements of each Jacobi matrix with Formula 11.

$$
S^{m} \rightarrow S^{m-1} \rightarrow \ldots \rightarrow S^{2} \rightarrow S^{1}
$$




$$
[J]_{h j}=\frac{\partial e_{k q}}{\partial \omega_{i j}^{m}}=\frac{\partial e_{k q}}{\partial n_{i q}^{m}} \times \frac{\partial n_{i q}^{m}}{\partial \omega_{i j}^{m}}=S_{i h}^{m} \times \frac{\partial n_{i q}^{m}}{\partial \omega_{i j}^{m}}=S_{i h}^{m} \times \alpha_{j q}^{m-1}
$$

(3) Obtain $\Delta \omega$ with Formula 4

(4) Repeatedly calculate the sum of squared errors with $\omega+\Delta \omega$. If the new sum is less than the sum in the first step of calculation, divide $\mu$ by $\theta(\theta \geq 1)$, and $\omega=\omega+\Delta \omega$, turn to the first step; otherwise, multiply $\mu$ by $\theta$ and turn to the third step. While the sum of squared errors decreases to certain target error, the algorithm is considered to be converged.

Compared with traditional BP and other improved algorithm, L-M optimization algorithm has less iterations, faster convergence rate and higher accuracy. So L-M optimization algorithm has favorable superiority in BP network learning.

\section{Experimental Verification}

Experimental data come from database of 60 scientific and technical major teachers of Wuhan Railway Vocational College of Technology (referred to as WRVCT), and Wuhan Polytechnic (referred to as WP) and Shanghai Hubei Communication Technical College (referred to as HCTC) and 1800 students from the same three universities. Limited to paper space, the evaluation of intermediate results is omitted here, only providing parts of (secondary) evaluation results and final comprehensive evaluation results, see table 1 and table 2, and in which the average evaluation results of the teachers of each vocational college.

As for the performance of the presented algorithm, this thesis also realizes the application of the ordinary BP neural network [3] and comprehensive fuzzy evaluation [4], evaluation performance of different algorithms is shown in Table 3. In table 3 evaluation results of training effects of different students are selected and compared with artificial evaluation to calculate the evaluation accuracy. And the calculation platform as follows: hardware is Dell Poweredge R710, in which processor is E5506, memory 2G, hard disk 160G; software platform is Windows XP operating system, C programming language environment.

Table 1. Evaluation Results of First grade Indicators and Final Evaluation

\begin{tabular}{cc|c|c|c|c|c|c}
\hline & $\begin{array}{c}\text { Teaching } \\
\text { Management }\end{array}$ & $\begin{array}{c}\text { Course } \\
\text { Construction }\end{array}$ & $\begin{array}{c}\text { Teaching } \\
\text { Conditions }\end{array}$ & $\begin{array}{c}\text { Teaching } \\
\text { Process }\end{array}$ & Teachers & $\begin{array}{c}\text { Teaching } \\
\text { Effects }\end{array}$ & $\begin{array}{c}\text { Final } \\
\text { Evaluation }\end{array}$ \\
\hline WRVCT & 4.021 & 4.213 & 3.589 & 4.013 & 3.654 & 4.225 & 4.018 \\
WP & 4.213 & 4.354 & 4.331 & 4.278 & 4.361 & 4.541 & 4.378 \\
HCTC & 4.332 & 4.331 & 4.439 & 4.341 & 4.561 & 4.651 & 4.471 \\
\hline
\end{tabular}

Table 2. Evaluation Performance Comparison of Different Algorithms

\begin{tabular}{lccc}
\hline & $\begin{array}{c}\text { Algorithm in This } \\
\text { Paper }\end{array}$ & $\begin{array}{c}\text { Ordinary BP Neural Network } \\
\text { Algorithm }\end{array}$ & $\begin{array}{c}\text { Comprehensive Fuzzy } \\
\text { Algorithm }\end{array}$ \\
\hline Accuracy Rate & $93.77 \%$ & $82.43 \%$ & $68.33 \%$ \\
Time & 13 & 376 & 14 \\
Consuming(S) & & & 14 \\
\hline
\end{tabular}

\section{References}

[1]. Huang Puquan. On curriculum ideals and curriculum evaluation. Journal of South China Normal University (Social Science Edition), Vol. 14 (2011) No. 6, p. $55 \sim 61$.

[2]. Yang Jing. University education specialized university network teaching reform and Research of the chorus at Network Age. Journal of Theory Research, Vol. 14 (2010) No. 19, p. 238 240.

[3]. Hongbo Mu, Dawei Qi, Mingming Zhang. University Base teaching Evaluation with Flaw Based on BP Neural Network. Journal of Convergence Information Technology, Vol. 8 (2013) No. 1, p. $307 \sim 315$. 
[4]. Mingkuai Zhou, Rongzhen Fan. Study on the Extraction Algorithm of Digital Watermarking Based on Neural Network. International Journal of Digital Content Technology and its Applications, Vol. 7 (2013) No. 1, p. $460 \sim 468$.

[5]. Xinmei Guo. The Study and Implementation of Back Propagation Neural Network Technology. International Journal of Digital Content Technology and its Applications, Vol. 7 (2013) No. 2, p. $694 \sim 702$.

[6]. Zhao Hongguang. The study on the Education Method of the Chorus courses in Teachers Training Colleges Zhao Hongguang. Journal of Nanjing Arts Institute Music \& Performance, Vol. 14(2009) No. 2, p. $80 \sim 84$.

[7]. Zhang Hongwei. The Report on Experiment of Teaching the Course of Chorus and Conducting and Its Curriculum in Normal Institution.Journal of Qujing Normal University, Vol. 14 (2008) No. 4, p. $115 \sim 119$.

[8]. Yu Jinghuai. Study on Evaluation System for University’s Off-campus Practice Teaching Bases (PTBs). Journal of Education Research,Vol. 21 (2011) No. 7,p. 34 29. 